\title{
Coronation's Disturbing Effect: Even After they Recuperate - The Sense of taste and Smell did not Return to Some of the Adhesives
}

\section{Shimon Shatzmiller*}

Department of Biological Chemistry, Ariel University, Israel

*Corresponding Author: Shimon Shatzmiller, Department of Biological

Chemistry, Ariel University, Israel.

A Wall Street Journal study claims that a high percentage of recovering Corona researchers already claimed that the loss of taste and smell is one of the symptoms of those infected with the virus. Some patients experience this sensory loss along with other symptoms, such as high fever, cough, or shortness of breath. According to a study published in the Wall Street Journal, some patients have recovered from Coronavirus SARS-COV-2 and most of its many effects on the human body. But have reported that their sense of taste and smell has not returned to this day.

For more updates and submitting your stories - go to the news Facebook page

Many of the corona's effects on the human body, even in the long term, are still unknown. According to a study, it was found that the loss of taste and smell did not improve in some patients, even after all other symptoms disappeared. "Doctors claim that some healers may not feel taste or smell in the future" it said.

A study published in late April in Europe claimed that out of 417 corona patients tested, whether mild or severe, $88 \%$ reported loss of taste and smell. According to the data, most of those reports failed to smell or feel good even after recovering from other symptoms.

About a quarter of the reporters reported that the senses returned to them about two weeks after the rest of the corona symptoms disappeared. The study also found that women in corona illness lost, much higher than men, their senses of taste and smell.
Received: July 11, 2020

Published: August 25, 2020

(C) All rights are reserved by Shimon

Shatzmiller.
However, the researchers made it clear that further research and examination of more cases is needed to decide for how long recovered people from Corona lose their senses.

The effects of loss of sense of smell have also been studied, and are broader than morning coffee or pizza flavor. Loss of senses often leads to weight loss and health problems. Also, patients will not be able to smell a fire or gas leak if needed. Also, a study conducted at the University of Norwich in England found that loss of taste and ability to smell caused patients with depression, anxiety and loneliness.

"The loss of taste and smell is caused by receptors that prevent the information from reaching the brain," one doctor explained. "It may be a natural response of the body to the virus. If it doesn't work, people may be more sick".

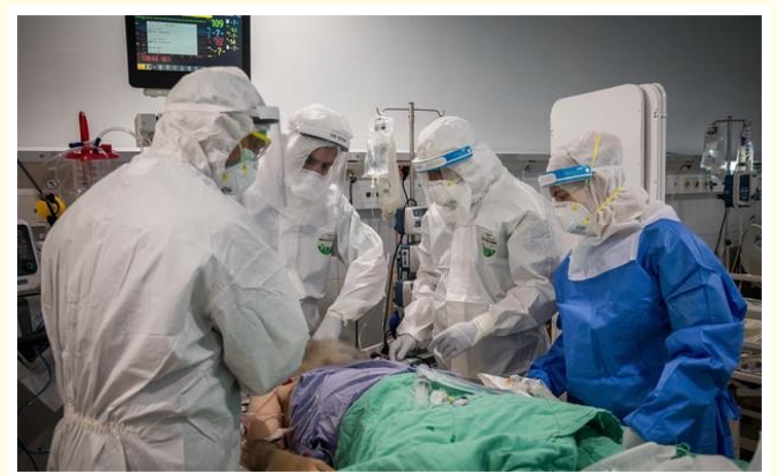

Figure 1: Operation room in corona times. 
Today, in many parts of the world, the behavior of the virus and its damage to the human body are better understood: In Short, after infiltration, the virus penetrates the respiratory system, into the lungs and damages the delicate lung tissues that lose their ability to provide oxygen to the body. And from it to the circulatory and intestinal and intestinal organs. From there it comes to the brain and there causes neurological injuries like loss of taste and smell. But not only that. The virus can cause the immune system to damage the brain tissues and lead to death.

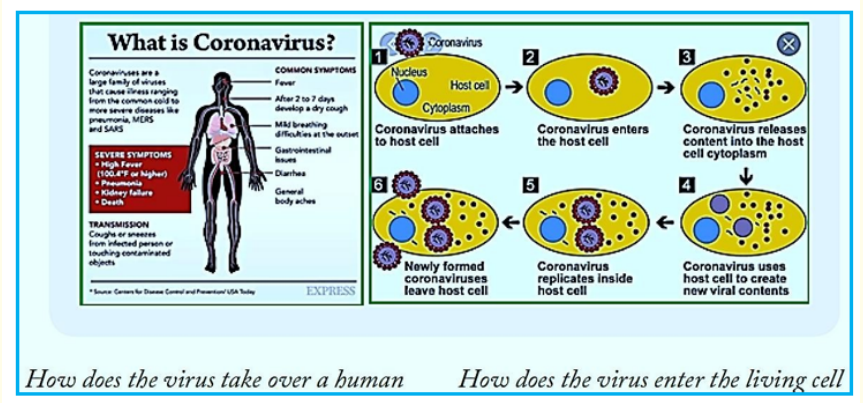

Figure 2

Intestinal microbes contribute to human health through roles in polysaccharide decomposition, nutrient absorption, inflammatory responses, gastric permeability and bile acid change. Many studies have shown that disruptions in the relative proportions of gut microbial populations may contribute to weight gain and insulin resistance, for example. This includes changes in microbes and the association of Firmicutes with weight gain Bacteroidetes and possible modifications in microbe-producing microbes, such as Bacilli bacteria. It has also been shown that the attenuated Archaea may contribute to metabolism and host weight gain. However, most studies are performed using fecal or colon samples and may not represent the active small intestine in metabolism. Studies primarily in rodent models are beginning to elucidate the mechanisms by which gut microbes contribute to DM and obesity for instance, but still have to be studied before we can begin accessing targeted therapies.

Coronaviruses are RNA viruses that are prickly as predictions On its wrapping surface and giving it a crown appearance. Hence the term coronavirus virus (Singhal, 2020). Phylogenetic diversity Studies have highlighted that SARS-Cov-2 shares 79\% nucleotide sequence Identity with another virus from the same SARS-Cov family which Caused severe epidemic in 2002-2003 which resulted in 8000 cases in 26 States. In addition, SARS-Cov- 2 also presents Sequence identity of $96 \%$ and $89.6 \%$, for the envelope and nucleocapsid Proteins respectively with SARS-Cov. Middle East respiratory syndrome or MERS disease in 2012 was as well Caused by another coronus virus called MERS-CoV. All these viruses Use the ACE-2 receptor on cellular entry. Interestingly, ACE-2 receptors are also expressed Kidney and digestive system, tissues known as SARS-CoV limitations. Previous evidence has suggested That viral RARS of the SARS virus could be detected in respiratory secretions And excrement of some patients after the onset of the disease for more than One month but a live virus could not be fostered after a third week (Chan., et al. 2004). Now, the latest report also suggests that SARSCov2 RNA can Will be detected in the stools of some Covid-19 patients (Wu., et al. 2020). This Along with the fact that some patients have diarrhea Indicates a distinct possibility of involvement of the gut axis and may be the gut microbiota.
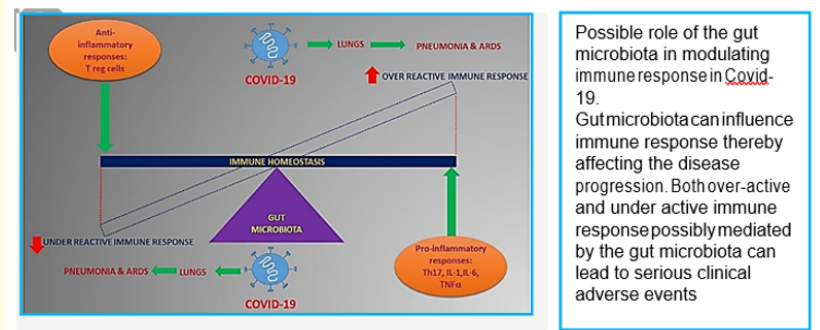

Figure 3: Possible role of the gut microbiota in modulating immune response in Covid-19.

Six months from the COVID-19 epidemic, we are still learning what the disease can do. Now there are detailed reports of brain diseases that occur in people with relatively mild lung disease, in those suffering from human beings and in addition to recovery.

One key thing we see is that the severity of lung disease does not always match the severity of neurological disease. Only mild lung disease does not protect against potentially serious complications.

When it comes to the brain and nerves, the virus seems to have four main groups of effects: 
- Confused state (called delirium or encephalopathy), sometimes with psychosis and memory disorder.

- Brain inflammation (known as meningitis). This includes a form that shows inflammatory lesions - acute disseminated meningitis (ADEM) along with the low oxygen effects in the brain.

- Blood clots leading to stroke (including in younger patients).

Possible damage to the nerves in the body, which causes pain and numbness (for example, in the form of post-infection GuilinBarra syndrome, in which your body's immune system attacks the nerves). Today these patterns of effects look similar across the world. Some of these diseases are fatal, and for those who survived, many will carry long-term consequences.

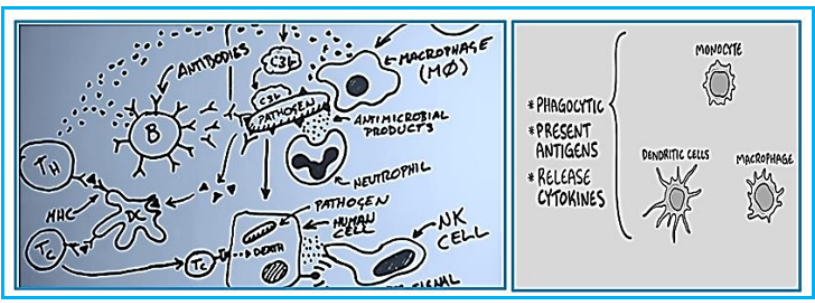

Figure 4: The Complexity of the Immune response.

This raises an important question: Will COVID-19 be associated with a major epidemic of brain disease, in the same way that the flu epidemic of 1918 was associated (to be admitted with little certainty) to the epidemic of encephalitis (sleep disease) that prevailed until the 1930s? It's hard to say at this point - but here's what we know about the effects of the virus on the brain so far. All of these effects on the brain and nervous system have the potential for long-term damage and can pile up in a person. But we need to know more about what's going on in people's nervous systems before we can accurately predict any long- term impact. As COVID-19 spread throughout the country, great attention is paid to the devastating effects of the virus on the lungs.

But doctors are learning how the virus can affect other organs, including the brain.

Some patients with COVID-19 had neurological symptoms, including headache, loss of taste and taste, hallucinations, vivid dreams, meningitis and even seizures or stroke.

Acute respiratory distress syndrome (ARDS) as a result of SARSCoV-2 infection was associated with encephalopathy, prominent confusion, and colorectal tract signs. Two of 13 patients who underwent cerebral MRI underwent one acute ischemic stroke. No data are available to determine which of these features were due to disease-related encephalopathy, cytokine or drug effect or withdrawal, and which features were specific to SARS-CoV-2 infection.

Brain inflammation due to invading microbes via the gut-brain doute was suspected to cause neurological brain damage some time ago.

The inflammatory hypothesis

The intrinsic model

Currently, there are two models of the inflammatory hypothesis of $\mathrm{AD}$, intrinsic and uniform. The essential inflammation model is responsible for completeness 'blood-brain barrier' (BBB) restricting entry of neurotoxic immune molecules and systemic lymphocytes to the brain. As a consequence, the brain glial cells can generate a local and complete innate immune system when challenged by foreign agents. Historically, neuroinflammation has largely been viewed as being a downstream consequence of the amyloid hypothesis, the presence of amyloidogenic peptides results in the activation of a microglia that starts an inflammatory cascade and the release of neurotoxic agents that can cause neuronal degenerative changes. GWAS currently suggests innate immune genes as a risk factor and supports a primary role for the inflammatory elements of $\mathrm{AD}$ pathology through inappropriate activation of complement system in association with $\mathrm{Ab}$ plaques and neurofibrillary tangles (NFTs).

\section{Curing Alzheimer's - Is there any potential Remedy?}

New approaches in $\mathrm{AD}$ drug discobvert

Once inflammation is active, it immortalizes its self greatly. These inflammatory cytokines migrate throughout the body and cause oxidative stress. Oxidative damage is the earliest event of Alzheimer's disease. Destruction of the fragile machinery of tissues and mitochondria, especially. In the brain, inflammation is used to stave off the use of tryptophan toward the production of anxiolytic chemicals such as quinoline, instead of serotonin and melatonin. They produce a reproduction collection of symptoms known as 


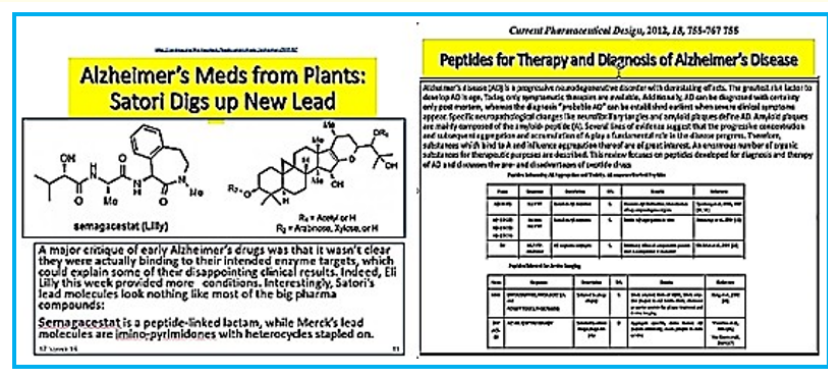

Figure 5: Innovative approaches in neurodegeneration drug discovery.

"sickness syndrome", which is marked by its overlap with "depressive" symptoms: fatigue, sleep disturbances, decline in social activity, mobility, sexual desire, learning, anorexia and anathonia. Psychiatric researchers have noted that patients with higher levels of inflammatory markers (such as CRP, reactive protein $\mathrm{C}$, are less likely to respond to antidepressants. In the same time, more likely to respond to anti- inflammatories, For example, AMP cases alter mammalian membrane properties or interact with its receptors to affect diverse cellular processes including cytokine release, chemotaxis, antigen presentation, angiogenesis, and wound healing. Today, we are beginning to see that much of the importance of AMP in mammals may be in their multifunctional role. However, growing evidence suggests that some AMP spaces can provide protection by an indirect mechanism and not just because they can kill bacteria. They can function as potent immune regulators, alter host gene expression, act as chemokines and induce chemokines, inhibit LPS or hyaluronan caused by proinflammatory cytokines, promote wound healing, and modulate dendritic or T cell responses. Immune response. In this way AMPs act as a bridge between innate and adaptive immunity. These functions favor resolution of infection and make inflammation potentially harmful, complementing bacterial direct action. Here we look at some of the essential functions. Of AMPs unrelated to their direct antimicrobial action.

Ultrashort SMAMPs (Synthetic Mimics Antimicrobial Peptide). Can be very instrumental in neurosurgery. We demonstrated that SMAMPs (mimicking 5 amino acids), which are based on some ascribed scaffolding as 1,4 -DHP and azine cross the BBB, and enter the stroma and parenchyma of the living brain. There is currently an immediate need to improve biomarker and psychiatric, developmental, traumatic, inflammatory, infectious and degenerative therapies. These, in whole or in part, are a significant social burden as a result of the increase in the number of people affected and the severity of the disease.

Lost productivity of the patient and his or her caregiver and the emotional and financial burden cannot be overstated. The need for improved health care, treatment and diagnostics are immediate. What a means to such an end is nanotechnology. Indeed, recent developments in health-care enabling nanotechnologies and nanomedicines range from biomarker discovery including neuroimaging to therapeutic applications for degenerative, inflammatory and infectious disorders of the nervous system.

The role of antimicrobial peptides (AMPs) and synthetic imitation of antimicrobial peptides (SMAMPs) in brain inflammation.

In particular, cationic peptides, such as the $\beta$-defensins, originally assigned to antimicrobial function, are now recognized as mediators of both innate and adaptive immunity. The following is supporting evidence to suggest that neuropathological changes associated with chronic central nervous system disease include abnormal expression and regulatory function of specific antimicrobial peptides. It is also suggested that these changes exacerbate the proinflammatory conditions in the brain that eventually strengthen the degenerative process.

How can antimicrobial surrogates contribute to neural degeneration research?

- Back inflammation; AMPs are anti-inflammatory.

- Old Foldamers as secretase inhibitors.

- Ept antiviral antimicrobial peptides held are not accumulated.

- Immunotherapy.

- The unique mechanism of secret-secretase inhibition. Notch function of GSI $\gamma$-secretase inhibitor.

- Aib has helped penetrate BBB for testing and medication.

The polypeptides mentioned above suffer from a significant disadvantage, namely their ability to penetrate the BBB and this from Protease digests them. Their features are far from LIPINSKI's 5 statements. 
In this respect, SMAMPS may be useful for treating neuritis. The many shortcomings of AMP are omitted by the design and synthesis of such surrogates. Antimicrobial peptides are very sensitive compounds and suffer from many drawbacks when intended to be applied by humans in their struggle against the microbes:

Development of Antimicrobial Peptides as Anti-Infective Drugs.

\section{Advantages disadvantages}

- Broad-spectrum activity (antibacterial, antiviral, antifungal) Discovery costs of synthesis and screening

- Rapid onset of killing Patent exclusivity for economic viability

- Cidal activity Systemic and local toxicity

- Potentially low levels of induced resistance Reduced activity based on salt, serum, and pH sensitivity

- Concomitant broad anti-inflammatory activities Susceptibility to proteolysis

- Pharmacokinetic (PK) and pharmacodynamic (PD) issues

- Sensitization and allergy after repeated application

- Natural resistance (e.g., Serratia marcescens)

- Confounding biological functions (e.g., angiogenesis)

- Among the many advantages, there are also drawbacks in which the hardest are instability in biologic environments (decomposition by enzymes) size of the molecule and difficulties to apply in high scale.

We would like to use the exploring of some leading SMAMPS as Neurodegeneration retarding compounds.

In addition to their antimicrobial role, AMPs also serve as important effector molecules in inflammation, immune activation, and wound healing. Antimicrobial peptides are needed to combat neuroinflammation antimicrobial peptides are part of the innate immune system of many organ systems, yet very little is known about expression and function in the brain. The antibacterial AMP in rats (a human LL-37 homologue) not only exhibits strong bacterial activity, but also functions as an attractant for immune cells. AMPs participate in brain immunity by stimulating cytokine production and activating glial cells and help protect brain cells by creating neurotrophic factors. AMPs are important components of innate central nervous system immunity that act to protect brain cells by inducing neurotrophic factors.
Anti-microbial peptides in neurons are major players in neurodevelopmental delay. $\beta$-Defensins have major tasks for protecting the brain and lobular parenchyma. AMPs Amylin (sugar metabolism) amyloid and others such as insulin (diabetes) play a major role in inhibiting the spread of inflammation and infection by their involvement in the harnessing of immune stimulants as follows: The human $\beta$-defensins (hBDs) are a highly conserved family of cationic antimicrobial and immunomodulatory peptides that are expressed mainly in epithelial cells in response to invasion by bacteria, fungi and some viruses. To date, the most widely studied in this family of peptides are HBD-1, -2 , and -3 . Expression of HBD-1 and 2 has previously been demonstrated in cultured microglia and human and human brain astrocytes. Unlike hBD-2 and -3, HBD-1 is constitutively expressed and is not regulated by pre-inflammatory factors.

The American Academy of Dermatology suggests to take the

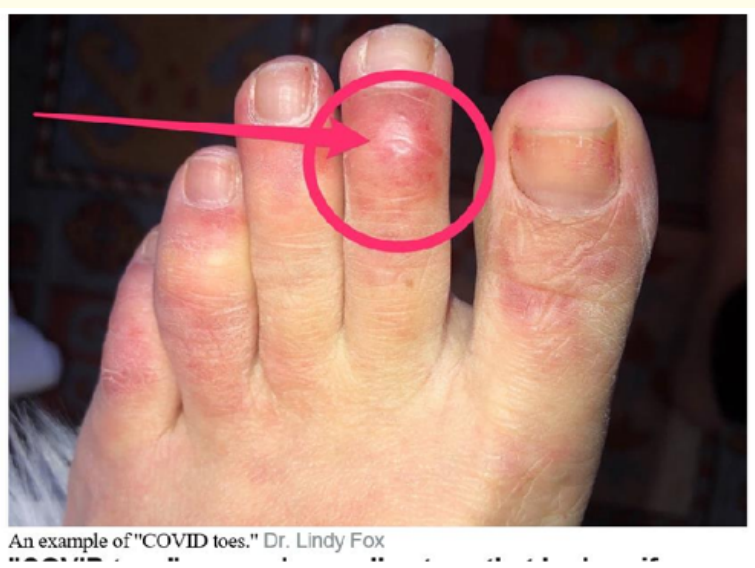

Figure 6: Corona Toes Test.

"corona toes test" as a simple additional test is the coronas diagnosis process.

Now, more than 300 studies from around the world have found a prevalence of neurological abnormalities in Covid-19 patients, including mild symptoms such as headaches, odor loss (anosmia) and tingling sensations (arcoparesthesia), to more severe outcomes such as aphasia (inability to speak), stroke and seizures. This is in addition to recently published findings that the SARS- 
COV-2 virus (COVID 19), mostly considered a respiratory disease, can also cause damage to the kidneys, liver, heart and almost any organ system in the body.

\section{Assets from publication with us}

- Prompt Acknowledgement after receiving the article

- Thorough Double blinded peer review

- Rapid Publication

- Issue of Publication Certificate

- High visibility of your Published work

Website: www.actascientific.com/

Submit Article: www.actascientific.com/submission.php

Email us: editor@actascientific.com

Contact us: +919182824667 